\title{
Fostering Quality of Reflection in First-Year Honours Students in a Bachelor Engineering Program Technology, Liberal Arts \& Science (ATLAS)
}

\author{
Pascal Wilhelm \\ University of Twente
}

This study focused on fostering the quality reflection displayed in semester self-evaluation reports (SERs) of first-year honours students in a bachelor engineering program Technology, Liberal Arts and Science (ATLAS). In a first pilot study, students' conceptions of reflection and educational needs regarding reflection were explored. Based on the results and relevant theory, an intervention was designed. Twentynine participants, not previously exposed to academic training on reflection before, received a Reflection Guide on how to write reflections in their SERs. Two online interactive lectures were provided as support. Quality of reflection in the SERs was assessed using a standardized rubric and quality scores in the intervention group were compared with scores of the student cohort of the previous academic year $(n=$ 33). Results showed that the intervention group reflected on a higher level than the comparison group. Perceived usefulness and value of the reflection method proposed were measured in both students and assessors. In general, both students and assessors were positive about the reflection method.

Keywords: reflection, higher education

\section{INTRODUCTION}

ATLAS University College Twente is a bachelor of science program in Technology, Liberal Arts \& Science that aims at educating the 'New Engineer' (Goldberg \& Sommerville, 2014). The program has embraced the concept of self-directed learning (Gibbons, 2002; Saks \& Leijen, 2014), meaning that students attain learning goals mostly in their own way. Semester goals (there are six semesters) define a developmental framework that allows students to gradually build their own academic profile as New Engineers. At the same time, the framework safeguards that all students reach the intended learning outcomes of the program as a whole. At the start of each semester, students write a plan in which they explain how they intend to reach the semester goals of their current semester. At the end of each semester, students write a self-evaluation report (SER) in which they reflect on their development and evaluate whether they reached the goals of the semester. The SER is input for a semester assessment meeting in which a group of assessors decides to what extent the students' self-evaluation can be justified. As part of this assessment, students receive feedback on the quality of reflection and self-evaluation displayed in their SERs. As a requirement to pass the semester, quality of reflection and self-evaluation needs to be up to par.

Reflecting and self-evaluating are considered important skills by the program, both for the students' learning and their role as New Engineers in society. New Engineers typically work interdisciplinary, meaning that they need to be able to quickly familiarize themselves with new fields of expertise to be a linking pin in interdisciplinary teams. Actively monitoring what one knows and what still needs to be 
learned through continuous reflection and self-evaluation is therefore of seminal importance. Life-long learning characterizes the professional practice of New Engineers and reflection is regarded an important skill in this respect (Rogers, 2001, see also Yost et al., 2000).

Skills in reflection do not come naturally, but can be trained (e.g. Gün, 2011; Kori et al., 2014; Russel, 2005). Up until the moment of the current study, the ATLAS program offered support to first-year students in writing their SERs by providing two pages of written instructions and one 1.5-hour lectorial (interactive lecture, similar to a workshop) on the nature of reflection and self-evaluation. However, the effect of this support, especially with respect to reflection, was not reflected in their SERs. Teacher-assessors generally noted the poor quality of reflection displayed in the SERs, but also appeared to use different criteria for assessing the SER reflections. Typically, the assessment manuals for assessors contained no information about criteria to use in providing feedback on the quality of reflection. Moreover, students did not seem to be aware what was expected of them with respect to reflection. These observations formed the motive for this study, the underlying problem being unclarity surrounding what constitutes proper reflection, both on the students' and the teachers' side. Without such clarity, the conditions needed to develop reflection skills are at least suboptimal. Typically, quality of reflections in the SERs remained stable throughout the program.

The research questions of this study were 1) What would be an appropriate reflection method for firstyear ATLAS students that includes both support measures and feedback criteria? 2) What is the effect of an intervention targeted at fostering quality of reflection? and 3) What is the level of perceived usefulness and value of the proposed reflection method on which the intervention was bases for students and teacherassessors?

Five studies were conducted. First, ATLAS students' current understanding of reflection and their educational needs in this respect were explored using an online survey with open-ended questions (Study 1). Based on the results of Study 1 and a literature review on interventions to foster reflection, an intervention was designed, implemented and evaluated (Study 2). In this study, the effect of the intervention was tested in a between-subjects design by comparing the quality of reflection displayed in the SERs of a first-year intervention group (Class of 2023) with the first-year cohort of the academic year before (Class of 2022). In a short post-intervention survey, students' perceived usefulness and value of the reflection method was measured to assess whether the intervention fitted their needs (Study 3). Parallel to this, assessors' initial feedback criteria to assess the level of SER reflection were studied to evaluate the alignment of their own criteria with the criteria proposed in the intervention method (Study 4). Assessors were then exposed to the reflection method applied in the intervention group and stimulated to use this information in providing feedback on the SERs. After the SER assessment of the Class of 2023, assessors were asked to fill out a short survey to measure perceived usefulness and value of the intervention method from their perspective (Study 5). These data would indicate to what extent implementation of the reflection method would be welcomed by the teachers-assessors in the near future.

The principal researcher designed and delivered the intervention, coded and analysed the data.

\section{Theoretical Framework}

Van Beveren et al. (2018) made an inventory of the purposes attached to reflection in higher education. Their research shows that what is considered "reflective" in higher education has many faces and the teaching of reflection shows a broad diversity of educational values and purposes. According to Fook et al. (2006, p. 4) the scholarship on reflection can be characterized as "a messy and complex field in which traditional disciplinary boundaries and shared criteria for academic rigor do not always apply." Clarà (2015, p. 261) stated that the concept itself has become "unanimously recognized in the field to be ambiguous."

Thus, the question what can be considered reflection seems difficult to answer. Its purpose in higher education does not stem from a unified idea, but is most likely operationalized by educators themselves, most probably with a limited view on the theory that is available. This is not necessarily a bad thing, reflection may be used for different purposes and educational programs can have different purposes with reflection. 
Despite the apparent fuzziness of the concept, Poldner et al. (2011) were able to identify the commonalities in reflections definitions. First, reflection is always directed at something, for example, a thought, experience or feeling and this 'object' of reflection can be more or less specific. Secondly, reflection is considered to be part of a cyclical process of learning that involves several stages and, thirdly, it is generally assumed that reflections can differ in strength. Fourthly, emotional involvement is, next to the cognitive aspect, also part of reflection. Lastly, commitment towards newly formulated intentions or ideas can be considered as an element of reflection.

The focus of this study is on written reflection in the SERs of the students, meaning it relates to what the students learned. Their assignment is to reflect on their "academic, professional and personal development" (ATLAS, 2020). The purpose of reflection in ATLAS becomes clear from the adopted educational approach of self-directed learning. In this respect, the ATLAS starting document (Ruijter \& Krab-Hüsken, 2012) states that "Self-directed learning in ATLAS means students learn for understanding and make conscious choices regarding both process and content. In order to do that, students need selfunderstanding and insight in their learning strategy and motivation. Owning their learning means they know what to learn, they know why they learn it, are capable of making the required choices, and reflect on their learning." So, reflection in ATLAS relates to what students learned. This is still a broad conception, but reflection needs to be distinguished from self-evaluation. This pertains to assessing learning performance against certain standards. So, reflection is not about whether students have reached certain pre-set academic (semester) goals, but what they took from their learning experience on a higher level.

ATLAS decided to ask students to reflect on their academic, professional and personal development. The SER writing instruction for semester 1 students (Class of 2023) state: "In the first part of your SER, you reflect on your academic, professional and personal development in the semester. Academic development concerns the course-related content, for example reflections on the theoretical knowledge you acquired. Professional development is about your skill development, reflections on any skills ranging from more domain-specific skills like programming to more domain-general skills like leadership - and collaboration skills. Personal development relates to how certain experiences in the semester have, for example, affected your beliefs, attitudes, norms, values, priorities and behaviours. You will receive feedback on the level of reflection displayed in your SER, as part of the "Feedback on the SER." Thus, it is clear that the reflections do not concern academic performance (self-evaluation), but are about their development in three broad, and possibly overlapping, areas. Moreover, students themselves decide what the reflect about in these areas.

Reflective ability is not a fixed trait, but it can be trained (e.g. Gün, 2011; Kori et al., 2014; Russel, 2005). However, reflection is considered challenging for students and, for different reasons, support is needed (Abou Baker El-Dib, 2007; Lee, 2005). For example, reflection requires creative thinking and seeing alternatives, which might be challenging for students (e.g. Leijen et al. 2012). Obviously, it is not enough to tell students to "go and reflect" (Welch, 1999).

Kori et al. (2014) reviewed several studies that aimed at supporting reflection in technology-enhanced learning environments. Several support measures were identified, ranging from videos, blogs and portfolios, to prompts and guiding questions, and peer/teacher interactions. Their research showed that these measures all have their benefits, but that this depends on the type of learning environment and the specific reflection activity. The authors also state that measures to evaluate the effect of the support measures in the studies they included in their review lacked in terms of validity and reliability.

For the underlying study, we based our intervention on the work of Ash and Clayton (2004) on articulated learning. On the one hand, their approach aligns with what Poldner et al. (2011) considered to be common to reflection definitions; 1) reflections are directed at something, 2) are part of a cyclical process, 3) can vary in strength, 4) include an affective component and 5) include an intention to change based on newly acquired insights. Also, there is evidence that their approach can foster quality of reflection (Ash et al., 2005) as measured with rubrics for assessing quality (or strength) of reflections. The rubric that was developed for this study was further inspired by Kember et al. (2008). Other means to assess the quality of reflection can be found in Dyment and O' Connell (2011). 
On the other hand, their approach to support reflection is straightforward, it makes use of scaffolding questions to structure student reflections and stimulates students to think deeply about their object of reflection. Peer/teacher interaction and feedback cycles (Ash et al., 2005) might add to the training effect, but in this case we decided not to add more to the intervention group' workload than strictly necessary because of the Covid-19 situation (the intervention was part of their curricular work). However, to increase the impact of the intervention, examples of reflections of different quality levels were provided, with an explanation of why, according to specific criteria, the example would fall into a certain reflection quality level category. This add-on is based on the well-established effect of learning from examples (see e.g. Hoogerheide \& Roelle, 2020).

\section{METHODS AND RESULTS}

\section{Study 1: ATLAS Students' Understanding of Reflection and Their Educational Needs Participants}

In total fifteen ATLAS students (eight females) were personally approached by email by the main investigator in the academic year 2019-2020. They were informed about the aim of the study and asked if they wanted to receive an online questionnaire. Among them, there were five students in their first year (two females), five in their second year (three females) and five in their third year (three females). Initially, all agreed, but in the end all but one student responded to the questionnaire, so the total number of participants was fourteen. Since the study was conducted in the context of a university program as part of a university teaching qualification trajectory it was not deemed necessary to report the study to an Ethical Committee. The main researcher took appropriate measures to follow the university's ethical guidelines for sound research behavior (see Procedure sections).

\section{Materials}

An online questionnaire with open-ended questions using the Google Forms platform was designed that contained the following questions:

1. Can you explain in your own words what is expected of you if you are asked, in you MTE and $S E R$, to reflect and self-evaluate? (discuss reflection and self-evaluation separately).

This question aimed at uncovering students' current understanding of reflection in ATLAS. Data regarding self-evaluation were not analysed for this study. The MTE is the Midterm evaluation in which students also need to reflect.

2. To what extent do you feel able to meet these expectations?

This question targeted at their belief in whether they felt able to reflect (self-efficacy).

3. What is your opinion on the educational support you get in learning how to reflect and selfevaluate in your MTE and SER? (discuss reflection and self-evaluation separately).

This question targeted to what extent participants felt supported in developing reflection skills. Data regarding self-evaluation were not analysed for this study.

4. Do you have any tips for the ATLAS program regarding this support? If you wish to share other opinions, ideas or tips concerning the topics of reflection and self-evaluation in ATLAS, you can do that here.

This question tapped into any ideas they might have for the educational support needed (if any, of course).

\section{Procedure}

The online questionnaire was provided via a link in an email. Students first read about the aim of the study, were informed that they voluntarily participated, that they could terminate the questionnaire at any moment, and that their data would be treated confidentially and processed anonymously. They actively agreed on these terms by clicking a button (informed consent). Students could indicate whether they wanted to be informed about the results after the study was finalized by providing an email address (later, it was 
decided to offer the results of the overall study to the entire ATLAS community). The questionnaire remained available for two weeks after which it was closed.

Data Analysis

Qualitative data analysis was used. First, responses were segmented into meaningful units. Then, meaning was extracted from these units and categories were made, also for further quantitative analysis (frequencies).

\section{Results}

Students' Current Understanding of Reflection

A dominant response to the question that targeted students' understanding of reflection was that reflection involved "looking back" to see what was learned and how things could be improved, still very much linked to the expected academic performance. Nine students responded in this or a very similar way. A typical response would be: "I have to look back on what I experienced and learned in the courses and reflect on how that has helped me reach certain learning goals for example, what was good and bad, what I learned and what I would like to improve on." Another example is: "I should look back at my performance and achievements and seek the strengths and weaknesses that I encountered. From these points, I should find points of improvement and things that I should maintain."

For three students, the object of reflection could go beyond the expected performance, for example: "look back at the semester and look at yourself. It's about measuring yourself and your personal development to your own ideas and standards. In the MTE and SER, this is what goes beyond the academic stuff." One student, very eloquently, put it this way: "Reflection for me means to go through the semester in my head and describe my journey in terms of emotions, decisions made, approaches taken and perspective adapted. During this process, realisations about your character, educational approach and academic competencies/interests are revealed. The honesty towards oneself in these realisations is the learning aspect of reflection. I believe reflection is meant to focus on the lesson rather than the productive outcome. Reflection prepares oneself for situations and scenarios that might occur in the future, that are similar to the lessons encountered. By using realisations of your reflection, you might find yourself to alter your decision-making or critical thinking in these situations. Additionally, reflection functions for me as a way to assess myself harshly but honestly without other consequences than personal growth."

Two students mentioned that reflections are about challenges and obstacles encountered: "After this, I write a reflection: how did the course/project/... go, what problems occurred and how did I tackle those." The other student wrote: "giving an indication on how many (un)expected challenges have manifested themselves over the first half of the quartile."

Although the students were asked to discuss reflection and self-evaluation separately, it was sometimes hard to see what they were discussing. For example, one student stated: "It is expected of me that I look back carefully at the (academic) activities relevant for the semester goals at hand. We must critically evaluate our performance by reviewing feedback that was given on hand-ins. The hand-in + the feedback creates evidence, and using this evidence we must argue whether we have obtained the semester goals. " Another student mentioned: "... and critically reflecting upon this evidence to identify whether the semester goals were met, but also upon your personal growth over the course of the semester and what aspects require attention in the following semesters." Perhaps the wording of the question challenged the respondents. Another interpretation might be that these students have difficulty separating between reflection and self-evaluation.

\section{Students' Self-Efficacy Concerning Reflection}

Results were quite clear here, twelve students stated they felt able to meet the expectations, explicated by statements like: "quite capable", "very capable", "I am pretty good", "fair expectations" and statements the like. In this group, two students admitted they had some difficulty with reflection at the start of ATLAS. Still, two other students found "reflecting quite difficult", one stated "I have trouble with reflecting at times.“ 
One student stated: "it is quite hard to separate reflection and self-evaluation, since they often complement each other naturally. In some cases, the border between these aspects remains unclear, which makes it difficult to discuss them individually." This might indicate that some students indeed may find it difficult to separate between reflection and self-evaluation.

Three students pointed out that expectations concerning reflection were not always clear to them, also pointing to the role of teachers. One student stated: "There isn't a real "guideline"/way to measure how indepth the reflection should be, so it can be hard to estimate if you were thorough enough." Another student put forward: "I to be honest have not known to a large extent how to improve upon my reflective skills as the feedback contained barely any improvement points", supported by a third student: "I noticed that different assessors have different expectations. Therefore, I sometimes get positive feedback on my reflection part and sometimes negative feedback. This has confused me in the past and made me rethink about what is expected of me and what I should note down in the MTE/SER."

\section{Educational Needs}

Nine students suggested that, despite acknowledged educational efforts of teachers (both formal and informal), there is still a need for additional support with respect to reflecting. For example, one student stated that: "Could be explained a bit better as I have the feeling that many people are still unsure as of what is expected in this part." Another student put forward: "ATLAS tries to vaguely explain what it means to reflect. This could be a good thing as it leaves the concept open to personal interpretation, but this should only be left open after a while" and a third student stated "Moreover, the amount of questions that followed the presentations showed the unclarity regarding the separation of reflection and self-evaluation."Finally, a fourth student stated "Educational support for reflection is lacking I think." Three students express to be happy with the support, for example: "I believe the educational support for both reflection and selfevaluation has been sufficient. ATLAS teachers have been open to helping students learn and develop reflection and self-evaluation skills."

Three students expressed that the difference between reflection and self-evaluation was not clear; "To know what the difference is between reflection and self-evaluation is something I never really got and am still unsure if I genuinely get it." This confirms the previous suggestion on this topic.

One student felt that what really helped them was not in the support already provided; "Self-directed learning [workshops to guide students in their ATLAS learning process] did not teach me too much about learning how to reflect and self-evaluate. Talking with students and teachers did. Dynamic conversations on how to think instead of getting into a lecture might be something worth considering as in my experience it has been the more effective source in learning from these things. These conversations are born out of the initiative of students which makes it inconsistent over the study. Facilitating the process would be a lot more useful in my opinion."

Two other students commented that support seems to decrease after a while: "I know that at the time, this really helped me in clarifying what I have to do. Now that I am in my second year, I do think I know what is expected of me, but as I said, sometimes the feedback is very diverse and I am confused as to what exactly I need to do better/improve (how)." The other student stated that: "In the beginning it was alright, but I feel like the support heavily dropped during the third semester."

Two students stated that reflection involves quite a lot of practice: "I think this is a typical case of learning while doing" and "but I have come to realize that it mainly takes a lot of practice for both [reflection and self-evaluation]."

\section{Student Suggestions for Improvement}

The suggested improvements put forward are threefold. First, four students expressed the need for clear instructions, templates and examples; "Hand a clear, concise overview of how to write a SER (the guidelines for the MTE are clearer already). What is expected? How do we know whether it's good enough? If we would have a check-list, this would make things a lot easier", "Providing students with examples of good/bad reflections and self-evaluations could help in making clearer what it is exactly is and what is expected of students", "Try to make expectations clear to the students and make sure all assessors are on 
the same page on what fulfilling these expectations could looks like (in like a framework, not super strict obviously, because that would defeat the purpose of individualism in the program)", and "It might be nice to have some sort of template, example from previous students, or detailed writing guide for the MTE and SER focusing on how to segregate self-evaluation and reflection." In addition, the need for more feedback cycles is expressed by two students: "be way more time spend on reflection and self-evaluation classes, as this is something that should be practiced and feedbacked" and "Furthermore, the MTE and SER should be accompanied by feedback specifically addressing these concepts. Ideally, there should then also be an opportunity to hand in an improved MTE or SER that implements this feedback, rather than delaying it to following semesters." Finally, more educational interaction was suggested, for example two students suggested having more writing sessions; "Offering more SER writing sessions where atlas professors are present could be a nice idea. This could offer quick support to students that show a sign of struggle".

\section{Summary Main Result Study 1}

Students mainly define reflection in their SER as a process of looking back at their semester learning trajectory to see what was learned and how they could improve, still all very much linked to the expected academic performance. Although the majority of students claim that they are able to meet expectations regarding reflection, these expectations are still not always clear to them. For example, distinguishing between reflection and self-evaluation seems particularly challenging. More than half of the students state that additional support for reflection is needed. Clear instructions, templates and examples, feedback cycles and writing sessions that allow for interaction with peers and teachers were mentioned as solutions.

\section{DESIGN OF THE INTERVENTION: ATLAS REFLECTION GUIDE AND LECTORIALS}

Study 1 made clear that students tend to focus their reflections on their academic performance. In this, the distinction between reflection and self-evaluation becomes blurry, which is corroborated by several statements that point to confusion about the distinction between them. Although most students feel that they can meet expectations regarding reflection, the majority states to need additional educational support. Clear instructions, templates and examples, feedback cycles and interactive SER writing sessions were suggested.

Due to the Covid-19 pandemic, education went online during the study and the first-year students were under quite some strain already. Therefore, it was decided to keep the intervention low-key. A Reflection Guide was provided with clear instructions, scaffolding questions that served as a template to structure reflections and to stimulate students to think deeply about a reflection object, quality criteria (rubric), and examples of low to high quality reflections with fictitious examples that highlighted the criteria. These ingredients mostly aligned with the needs identified in Study 1, with the exception of including peer/teacher interactions and feedback cycles, for reason mentioned before. See the Theoretical Framework paragraph for the theory that inspired the design. The guide can be found in Appendix 1. The Reflection Guide was written to be self-contained as additional instruction to write the SER, but the content of the guide was also discussed in two non-mandatory online lectorials of approximately 1.5 hours each (delivered via the Conference functionality in CANVAS, the courseware management system used by the University of Twente). The first lectorial focused in the students' task of writing reflections in their SERs, the second one repeated the first (on purpose; very few students showed up the first time), but also elaborated on the quality criteria and the reflection examples. There was room for questions in both lectorials.

\section{Study 2: Studying the Effect of the Intervention Participants}

The intervention group consisted of 29 first-year ATLAS students in their first semester (Class of 2023). There were 14 females, $58.6 \%$ of the whole class was of native Dutch origin. For comparison, the first semester SERs of 33 students of the Class of 2022 were used (also 14 females, $60.6 \%$ of the whole class was of native Dutch origin). Students in both cohorts were admitted on the basis of the same admission criteria (see EER ATLAS 2019 - 2020 and 2020 - 2021). Students from both cohorts that failed their semester were excluded from analysis. 
Since the SERs are written in the context of a university program as part of a university qualification trajectory it was not deemed necessary to ask the students for their consent and to report the study to an Ethical Committee. However, the researcher contacted the contact person of the Ethical Committee to inform about the use of the SERs for research purposes. The advice was to ask students for permission. Therefore, students were approached via email to indicate whether they objected to the use of their SERs for the purpose of this study (passive consent). Students were informed that their data would be treated confidentially and processed anonymously. In addition, they were informed that the results of the overall project would be shared with the ATLAS community. No students had objections. The SER file of one student in the comparison group was corrupted and was not analysed.

\section{Materials}

To assess the quality of reflection displayed in the SERs of the students, a rubric was constructed based on the levels of reflection described in the Reflection Guide. There are four levels of reflection and the rubric also contains intermediate levels, see Table 1 below. The parameters that define the level of reflection are: 1) understanding of the critical incident in term of the student's learning (understanding dimension); 2) implication of the learning for other situations in which it might apply (transfer dimension); 3 ) the personal impact or - significance of the incident (personal dimension) and 4) the extent to which the incident has had implications for future goals or behaviours (future dimension). The highest level, which is not common according to Kember et al. (2008), also includes evidence of a fundamental change in perspective.

\section{TABLE 1 SCORING RUBRIC QUALITY OF REFLECTION}

\footnotetext{
Level 1: Non- reflection

The intended reflection shows no evidence of the student attempting to reach understanding of a critical incident or significant learning experience. The student is basically evaluating his or learning on a general level, the learning is not related to any other situation in which it might apply. The reflection is not personal, it could have been written by any student. No implications for future goals or behaviors mentioned.

Level 1+: the reflection scores higher on certain criteria, but not enough for the reflection to be fully classified on the next level.

Level 2: Understanding

A critical incident or learning experience is described to a certain extent, and the student is able to explain its meaning, but does not relate it to any other situation in which it might apply. Also, it does not become clear in what way the experience matters for the student personally, or how it affected future goals or behaviors.

Level 2+: the reflection scores higher on certain criteria, but not enough for the reflection to be fully classified on the next level.

Level 3: Reflection

A critical incident is described clearly, and the student is able to explain its meaning and how it relates to other situations in which it might apply. It is also clear how and why the experience has mattered to the student on a personal level.

Level 3+: the reflection scores higher on certain criteria, but not enough for the reflection to be fully classified on the next level.

Level 4: Critical reflection

All of the above (level 3), while it is also clear how the incident has affected the student's future goals or behaviors. In addition, there is clear evidence of a change in perspective over a fundamental belief.

Note: criteria relate to four dimensions: understanding, transfer, personal and future
} 


\section{Procedure}

SERs were retrieved from the program's data base. The principal researcher first identified the different reflections in the SERs and then assigned a level to each of them on the basis of the rubric. An excerpt was considered a reflection when 1) students apparently "looked inside", and apparently thought about an experience or 2) when the excerpt was headed with "reflection" (this was common in the intervention group because they were instructed to structure their SERs in separate reflection and self-evaluation paragraphs). Students in the intervention group were instructed to write three reflections, while students in the comparison group could write as many as they liked. In this group, when students wrote more than three reflections, the three highest-scoring reflections were taken as units of analysis.

\section{Data Analysis}

Level 1 reflections were assigned a score of $1,1+$ reflections were assigned with a score of 1.5 , level 2 reflections were assigned a score of 2, etc. Mean reflection level was calculated by averaging the scores of the three reflections with the highest individual scores. Difference in mean quality of reflection was tested with a T-test for independent samples. Possible effects of gender and nationality (Dutch/foreign) were controlled for.

\section{Results}

On December 13 2020, the reflection guide was provided to the Class of 2023 via CANVAS. December 14 , the first lectorial was delivered. Four students attended this session in which they first read the guide. The main question or concern was that it could be hard to identify a personal experience (finding a critical incident), for example since Covid-19 would have had a similar effect on everyone. One comment was that it felt quite concrete what was in the guide. One student asked if he could have formative feedback on his first reflection and another asked about how many reflections he should write (this was stipulated in the guide, though) and also asked if he could receive formative feedback. On January 4, 2021 the second lectorial was delivered, attended by 21 students. There were no questions about the guide in this session.

First, it must be noted that students in the comparison group delivered reflections that were assessible with the criteria in the Reflection Guide. This indicates that they, to a certain extent, had similar ideas about what reflection entails compared to the intervention group. Therefore, it seems fair to compare the groups.

Average level of reflection in the intervention group was 2.0 (SD: .56, range $1.17-2.67$ ). In the comparison group, this was 1.3 (SD: .30, range $1.0-2.17$ ). A T-test for independent samples showed this difference to be significant $(\mathrm{t}=-6.64, \mathrm{df}=60, \mathrm{p}<.001)$; compared to the students in the comparison group, students in the intervention group reflected on a higher level. To provide more insight into the effect of the intervention, see Table 2. As can be seen, across the three strongest reflections (SRs) found in the SERs of the students in the comparison and intervention group, variation in the intervention group is stronger, with fewer reflections at level 1 and 1.5 and more at level $2-3$. In the comparison group, no reflections at level 3 and onward were found, while in the intervention group, these were quite common. No effects of gender were found. In the intervention group, difference in reflection levels between Dutch students and foreign students approached significance favouring the Dutch students (means were 2.2, SD: .54 vs. 1.8, SD: .53, sign. $<.06$ for the Dutch and foreign students respectively), however these groups were relatively small (17 vs. 13 students, respectively). Average number of reflections per group was 5.5 (SD: 3.1) for the comparison group and 4.8 (SD: 4.9) for the intervention group. This difference was not significant. Apparently, students in the intervention group tended to produce more reflections than the minimum of three that was expected. 


\section{TABLE 2}

\section{PERCENTAGES OF REFLECTIONS AT DIFFERENT LEVELS IN COMPARISON AND INTERVENTION GROUP}

\begin{tabular}{|l|l|l|l|l|l|l|l|l|}
\hline & \multicolumn{7}{|l|}{ Reflection levels } \\
\hline & SRs & 1 & 1.5 & 2 & 2.5 & 3 & 3.5 & 4 \\
\hline Comparison group & T1 & 39.4 & 33.3 & 21.2 & 6.1 & - & - & - \\
\hline$(\mathrm{n}=33)$ & T2 & 54.5 & 36.4 & 6.1 & 3.0 & - & - & - \\
\hline & T3 & 90.9 & 9.1 & - & - & - & - & - \\
\hline & & & & & & & & \\
\hline Intervention group & T1 & - & 13.8 & 24.1 & 20.7 & 34.5 & 6.9 & - \\
\hline$(\mathrm{n}=29)$ & $\mathrm{T} 2$ & 13.8 & 27.6 & 31.0 & 13.8 & 10.3 & 3.4 & - \\
\hline & $\mathrm{T} 3$ & 34.5 & 31.0 & 17.2 & 13.8 & 3.4 & - & - \\
\hline
\end{tabular}

Note: SRs: three strongest reflections found in the semester 1 Self-Evaluation Reports of the participants (T1 - T3).

Reflection levels: 1 = lowest, 4 = highest (see also Table 1 ).

\section{Study 3: Perceived Usefulness and Value of the Reflection Guide - Students}

\section{Participants}

The ATLAS Class of 2023 (full cohort, $\mathrm{n}=32$ ) participated in this study. There were 16 females. In total 18 students (gender not specified) responded to the questionnaire. Since the study was conducted in the context of a university program as part of a university qualification trajectory it was not deemed necessary to report the study to an Ethical Committee.

\section{Materials}

An online questionnaire was constructed to measure the perceived usefulness and value of the Reflection Guide. The questionnaire consists of ten items that can be scored on the five-point Likert scale ((1) Totally disagree - (2) Disagree - (3) Neutral - (4) Agree - (5) Totally Agree). The items are as follows:

1. The information about reflection in the guide was clear to me.

2. I studied the guide thoroughly before I started writing my SER reflections.

3. The information about reflection in the guide was new to me.

4. Based on the guide, I altered the way I normally reflect on my learning.

5. After reading the guide I knew what I had to do to write my own reflections.

6. While I was writing my SER reflections, I regularly looked into the guide.

7. Based on the guide, I feel able to explain in my own words what distinguishes low-level from higher-level reflections.

8. The examples of low- and higher-level reflections helped me to write my own reflections.

9. The information in the guide did not support me in writing my reflections.

10. In general, I feel that the approach towards reflection as explained in the guide is valuable for my learning.

In a final, open-ended question stating "Do you have any suggestions for the reflection guide?" students could comment on the guide more in-depth.

\section{Procedure}

The online questionnaire was provided via a link in an email. Students first read about the aim of the study and then they were informed that they voluntarily participated, that they could terminate the questionnaire at any moment, and that their data would be treated confidentially and processed anonymously. They actively agreed on these terms by clicking a button (informed consent). The questionnaire remained available for one week after which it was closed. 
Data Analysis

After the scale date for item 9 was recoded $(1=5,2=4$, etc.), reliability of the scale was assessed using Cronbach's alpha and Guttman's Lamba 2. Means and standard deviations were calculated for the total scale and separate items.

\section{Results}

Reliability analysis showed that Cronbach's alpha was .67 (10 items), Guttman's Lamba 2 was .72, indicating that internal consistency of the scale was sufficient. Scale mean was high: 4.0 (SD: .46, range 3 -4.80 ), indicating a high reported rate of perceived usefulness and value of the Reflection Guide. The items that scored highest were item 8 (mean 4.5, SD: .98, The examples of low-and higher-level reflections helped me to write my own reflections) and item 9 (mean 4.4, SD: .70, The information in the guide did not support me in writing my reflections; mirrored item). The lowest scoring items were item 3 (mean 3.4, SD: 1.10, The information about reflection in the guide was new to me) and item 6 (mean 3.4, SD: 1.15, While I was writing my SER reflections, I regularly looked into the guide). Apparently, the students were neutral towards whether the reflection method was valuable for their learning (item 10, mean 3.7, SD: .96).

Eight students responded to the open-ended question with more specific comments. Three students made positive comments: "The guide was nicely formulated and quite helpful - especially for a first year", "While the reflection guide was useful in shaping the reflection, providing structure, and making clear which level it needed to reach", and finally "Otherwise, extremely helpful!"

Two students commented on the examples provided, one student stating that the examples exemplified different levels of reflection, but that there were no examples of reflections in each area of reflection (academic, professional and personal development). Another student suggested to have more examples, because the examples in the guide were quite specific and perhaps therefore difficult to "resonate with" ("Consider including multiple (2-3) examples of top tier reflection to demonstrate the reflection concepts applied in practise across a variety of student cases"). Two students commented on the topic of the reflections, stating that; "I didn't like that we had to focus on three different parts, because the parts will often overlap anyways (I think it would be a good suggestion, but not a requirement))" and "I would have preferred to be able to write more personal reflections instead of having to have (at least) one personal, one academic, and one professional." Other students also felt restricted; "you can give 3 of the fourth level reflections, but then you still have not really reflected on the whole semester, but on these 3 experiences" and "The reflection guide felt somewhat constricting at times for reflecting, it dampened some freedom. So maybe include that this is a suggestion, but your reflection does not have to adhere to all guidelines." Finally, one student suggested the following: "I would suggest to include a model about the reflection cycle (to see the SER reflexion in context; writing about is is just one part). My suggestion would be the ALACT model by Korthagen." Obviously, this student did not need the guide.

\section{Study 4: Feedback Criteria Used by Teachers to Assess Quality of Reflection Participants}

Ten teacher-assessors with various levels of experience (range $2-8$ years, three females) in providing feedback on the quality of reflection in SERs were asked to participate in this study. Six assessors responded to the call (expertise range $2-8$ years, all males). Three teacher-assessors had a background in social sciences/humanities and three had a background in natural science/mathematics. They had not been exposed to the Reflection Guide before they responded. Since the study was conducted in the context of a university program as part of a university qualification trajectory it was not deemed necessary to report the study to an Ethical Committee.

Materials

The following question was asked to the assessors in an email:

What criteria did you apply to give feedback on the level of reflection displayed in the SERs you assessed? In other words: what distinguished, in your view, lower from higher level 
reflections? For example, what kind of reflections would lead to a "Fail/No Pass" for the

"Feedback on the SER" and what kind(s) certainly to a "Pass?"

\section{Procedure}

Teachers-assessors (colleagues of the main investigator) were approached with an email explaining the rationale of the study. They were asked to reply to the email, writing their response to the question above in a few sentences (approx. 250 words). They were explained that here were no right or wrong answers, that only their personal approach to assessing quality of reflection of the SERs mattered. They were also explained that their responses would be treated confidentially and processed anonymously, and were asked to respond within one week.

\section{Data Analysis}

Qualitative data analysis was used. First, responses were segmented into meaningful units. Then meaning was extracted and units were categorized for further (quantitative; frequencies) analysis. A criterion was defined as a requirement or parameter that teachers considered relevant in assessing the quality of reflections.

\section{Results}

The responses of the teachers display a variety of criteria that are found relevant (range of different criteria per teacher $1-5$ ), but there is also a level of overlap. Up until this point in time, the ATLAS program did not ask assessors to use specific criteria to assess the quality of reflection, nor had the program agreed upon what is expected of the students when they were asked to reflect. It was assumed that students know how to reflect and teachers can recognize a proper reflection when they see one.

\section{Development}

What stood out in the responses is the requirement that the reflections address the students' development or learning across the semester. Five teachers made this point. For example, one teacher stated that students should "describe their longer-term goals and the link with the (personalised) semester goals", "describe how these semester-achievements fit with the longer-term goals" and "describe how the reached (personalised) semester goals helped them forward on their path to their longer-term goal", to conclude with "describe how new goals have emerged based on the work in this semester" Another teacher put it more simply by stating: "connects past experiences to future plans and ambitions" A third teacher stated: "highlights how the student developed throughout the semester" and a fourth stated: "where he started, to where he wanted to go, how did he experience the journey and how did he feel about reaching the end of the rainbow" The last teacher stated: "This means that they reflect upon their learning experiences in terms of the expertise and skills they have developed, how and what that means for their development."

\section{Writing Criteria}

Three teachers mentioned criteria that relate to the quality of the writing itself. For example, one teacher stated that reflections should be "well-written and well-structured, so the assessor can follow the line of thinking of the student." Another teacher stated that reflections should be "written in a clear, critical, and mature way." A third teacher stated that a reflection should contain a "clear, solid, substantiated story."

\section{Honesty}

Two teachers stressed that reflection should be honest, meaning that students are critical towards themselves. One teacher put it this way: The reflection is 'honest', in the sense that a student also pays attention to what was difficult, tiresome, not successful, etc., and addresses how (s)he dealt with that and learned from that. If all problems are attributed to external factors, or only described in general terms, this weakens the reflection." The other stated, quite honestly: "if I get the impression that the reflections in the SER are "fairy tales", I look for ways to show that it should be "not approved". I am quite allergic for the "bullshit factor"... " 


\section{Self-Evaluation vs. Reflection}

ATLAS students are asked to reflect and self-evaluate in the SERs. The "Feedback on the SER" addresses both the quality of reflection and self-evaluation. The latter addresses the question whether the student thinks he or she has reached the semester goals at honours level. Study 1 showed that several students do not sharply distinguish between the two. Some teacher responses also point into that direction, for example one teacher stated: "does the SER contain an insightful reflection on the evidence collected for the realization of the learning goals?" and another stated: "explains the reasons why she/he believes has achieved such goals at honours level while providing evidence from peers and experts, make it for a pass."

Some teachers seem to be well aware of the nature of reflections as explicated in the reflection guide. One teacher in particular mentioned that reflections should contain reference to "concrete experiences by themselves", an "analysis of the experience", a "description of the impact" and a "description of the consequences: how does the combination of experience plus reflection lead to changes (in behaviour, in plans, in expectations, etc)." Another teacher would give bonus points if a student showed to be able to "abstract from his/her experiences and formulate some more generic rules, models, etc."

\section{Summary Main Results Study 4}

Teachers use several feedback criteria for SER reflections. They mainly want student to reflect on their development and learning throughout the semester. Certain writing criteria (clarity, structure) are stressed and teachers appreciate honesty in the sense the students show they can be critical towards themselves. Some teachers do not seem to sharply distinguish between reflection and self-evaluation, just as several students do. A few teachers mention criteria that are aligned with what is described in the Reflection Guide.

\section{Study 5: Perceived Usefulness and Value of the Reflection Guide - Teachers Participants}

All five assessors (two females) that assessed the semester 1 SERs of the ATLAS Class of 2023 participated in this study (two male assessors also participated in Study 4). All assessors had received an assessment manual that contained the Reflection Guide as an appendix. In the assessment instructions, assessors were told that students had been instructed to use the guide in writing their reflections and that they could use the rubric with criteria it to give the "Feedback on the SER." No further instructions were provided. Since the study was conducted in the context of a university program as part of a university qualification trajectory it was not deemed necessary to report the study to any Ethical Committee.

\section{Materials}

An online questionnaire was constructed to measure the perceived usefulness and value of the reflection guide from a teacher-assessor perspective. The questionnaire consists of ten items that are scored on a fivepoint Likert scale ((1) Totally disagree - (2) Disagree - (3) Neutral - (4) Agree - (5) Totally Agree). The items are as follows:

1. The information about reflection in the guide was clear to me.

2. I studied the guide thoroughly before I started to write the "Feedback on the SER."

3. The information about reflection in the guide was new to me.

4. Based on the guide, I altered the way I normally give "Feedback on the SER."

5. After reading the guide I knew what I had to do to write "Feedback on the SER."

6. While I was writing the "Feedback on the SER", I regularly looked into the guide.

7. Based on the guide, I feel able to explain in my own words what distinguishes low-level from higher-level reflections.

8. The examples of low-and higher-level reflections helped me to write the "Feedback on the SER."

9. The information in the guide did not support me in writing "Feedback on the SER."

10. In general, I feel that the approach towards reflection as explained in the guide is valuable for our students' learning. 
In a final, open-ended question stating "Do you have any suggestions for the reflection guide?" assessors could comment on the guide.

\section{Procedure}

The online questionnaire was provided via a link in an email. Assessors first read about the aim of the study and then were informed that they voluntarily participated, that they could terminate the questionnaire at any moment, and that their data would be treated confidentially and processed anonymously. They actively agreed on these terms by clicking a button (informed consent). The questionnaire remained available for one week after which it was closed.

\section{Data Analysis}

After the scale date for item 9 was recoded $(1=5,2=4$, etc.), reliability of the scale was assessed using Cronbach's alpha and Guttman's Lamba 2. Means and standard deviations were calculated for the total scale and separate items.

\section{Results}

Reliability analysis showed that Cronbach's alpha was .74 (10 items), Guttman's Lamba 2 was .88, indicating that internal consistency of the scale was more than sufficient (mind though, there were only six respondents). Scale mean was high: 4.08 (SD: .41, range 3.50-4.60), indicating a high rate of perceived usefulness and value of the Reflection Guide. The items that scored highest were item 2 (mean 4.60, SD: .55, I studied the guide thoroughly before I started to write the "Feedback on the SER."), item 7 (mean 4.20, SD: .45, Based on the guide, I feel able to explain in my own words what distinguishes low-level from higher-level reflections) and 8 (mean 4.20, SD: .84, The examples of low-and higher-level reflections helped me to write the "Feedback on the SER."). The lowest scoring items were item 4 (mean 3.80, SD: .84, Based on the guide, I altered the way I normally give "Feedback on the SER.") and item 6 (mean 3.60, SD: 1.14, While I was writing the "Feedback on the SER", I regularly looked into the guide). Apparently, the assessors find the reflection method more valuable for the students' learning than the students themselves (item 10 assessors, mean 4.20, SD: .84, item 10 students, mean 3.72, SD: .96).

One teacher commented on the guide, suggesting to have a semester pre and post reflection on the students' intended profile as a New Engineer ("Reflection guide to identify shortcomings and a scaffold to reflect dynamically from micro to macro level that is nudging students to reflect in comparison to their imagined profile. It is just a suggestion, I am not sure if semester 1 would able to perform the following (if that's not the case already), pre and post semester reflection could the students to reflect systematically in view of their goal and intended profile.").

\section{CONCLUSIONS AND DISCUSSION}

The problem addressed in this study pertained to unclarity surrounding what constitutes proper reflection in the SERs, both on the students' and the teachers' side. The conditions needed to develop reflection skills were at least suboptimal, evidenced by reflections that were regarded as poor by teacherassessors, remaining stable throughout the program. Therefore, a reflection method was introduced and evaluated. The leading research questions of this study were: 1) What would be an appropriate reflection method for first-year ATLAS students that includes both support measures and feedback criteria? 2) What is the effect of an intervention targeted at fostering quality of reflection? and 3) What is the level of perceived usefulness and value of the proposed reflection method on which the intervention was bases for students and teacher-assessors?

With respect to the first question, students expressed the need for support in writing reflections in a pilot study. Instructions, templates and examples were mentioned, as well as feedback cycles and teacher/peer interaction. Based on these insights and theory, a Reflection Guide was designed that provided instructions (explanation), templates (scaffolding questions) and examples. Due to the workload of the students and Covid-19 the intervention did not contain extensive feedback cycles (e.g. formative 
teacher/peer feedback on written reflections) nor teacher/peer interaction. The intervention consisted of a Reflection Guide with instruction, criteria and examples, supported by two lectorials. In the SER writing instructions and the lectorials, the difference between reflection and self-evaluation was explained.

The evidence supports the hypothesis that the intervention has an effect. Difference in reflection level displayed in the SERs differed significantly between the intervention group and the comparison group. No effects for gender was found. In the intervention group, the Dutch students seemed to reflect on a higher level compared to the foreign students. Although this difference only approached significance and the sample sizes are, of course, low, it might still be worthwhile to consider this interaction in future studies.

Study 4 explored the criteria teacher-assessors use to give feedback on the reflections of the students. The data showed that teachers want students to reflect on their learning and development. Certain writing criteria and honesty are also valued. Some teachers seem to mix up reflection and self-evaluation, just as several students do. In general, the criteria applied are different from the ones proposed in the Reflection Guide. However, there is also overlap and some teachers mention criteria that are in the heart of the proposed criteria. In all, it is concluded that the "conceptual distance" between their own criteria and the proposed ones is not very large. This is deemed positive with regard to future implementation of the method.

Both students and teachers highly rated the usefulness and value of the reflection method. It was reported that the Reflection Guide was extensively used and especially the examples provided stood out in usefulness and value. This also exemplified by the critical comments made by the students about the examples provided.

In conclusion, despite being low-key due to Covid-19, the intervention was successful in its aim to foster the quality of reflection in the SERs of the students. In addition, both students and teachers valued the reflection method, implicating that there is support base for further implementation.

A few shortcomings of this study will now be addressed. Firstly, the study uses a between subject design to study the effect of the intervention. A pre-post control group design would have been a more valid test, because now we must assume that the intervention and comparison group were comparable in their initial reflective ability. However, splitting the Class of 2023 in two to create a control and comparison group was not deemed ethical because students might profit from the intervention in terms of study progress (a SER rewrite is needed when the quality of reflection is not up to par). Moreover, collecting base-rate information was deemed inappropriate because of the serious workload the students had. On the other hand, the comparison and intervention group were comparable in terms of gender distribution, Dutch/foreign nationality distribution and ATLAS admission criteria. Of course, the relevancy of these characteristics for reflective ability is not known (in fact, results showed gender and nationality were unrelated to mean level of reflection quality) and measures that could predict reflection ability were not taken, but overall it is assumed the comparison was fair.

Secondly, the reflections in the SERs were identified and coded by the main researcher. Although the coding rubric is deemed elaborate and insightful, a certain expectation on his hand could have influenced the results. Involving a second coder to assess the interrater reliability of the coding protocol would have solved this problem. Lack of resources and workload of teachers were the main reason for not doing this.

Thirdly, the data for Study 1, 2 and 3 were not linked to the individual participants. Instead, for every study a new sample was taken. For example, if we would have had one sample that participated in every study, we could have seen whether students who made extensive use of the guide were also the ones that produced the best reflections. However, for study 1, we needed a broader perspective on the educational support. Moreover, by that time, the Class of 2023 had no experience yet with reflection support in ATLAS, which would have produced very superficial information compared to including second and third years in the study as was done now. In addition, we expected that finding students who would participate in all three studies would have led to lower sample sizes than we had now.

Further research and development could focus on 1) creating common ground amongst teachers on what reflection in ATLAS should entail, 2) based on this common ground, adapt and further refine the reflection support methods, including criteria for good quality reflections, and 3) testing the effect of a more elaborate intervention including feedback cycles and peer/teacher interaction.

A student from the Class of 2023: 
Thank you for the [reflection] guide, it was extremely useful as a crash course for demonstrating the reflection skills required for the SER. Please do feel free to reach out if you have any further questions or if I could be of assistance!

Warm regards,

\section{REFERENCES}

Abou Baker El-Dib, M. (2007). Levels of reflection in action research. An overview and an assessment tool. Teaching and Teacher Education, 23(1), 24-35.

Ash, S.L., \& Clayton, P.H. (2004). The articulated learning: An approach to reflection and assessment. Innovative Higher Education, 29, 137-154.

Ash, S.L., Clayton, P.H., \& Atkinson, M.P. (2005) Integrating Reflection and Assessment to Capture and Improve Student Learning. Michigan Journal of Community Service Learning, 11(2), 49-60.

ATLAS. (2020). Writing instructions ATLAS SER_S1S3S5 2020-202. Internal education document.

ATLAS EER (Education and Examination Regulations) 2019 - 2020. Internal education document.

ATLAS EER (Education and Examination Regulations) 2020 - 2021. Internal education document.

Beveren, Van, L., Roets, G., Buysse, A., \& Rutten, K. (2018). We all reflect, but why? A systematic review of the purposes of reflection in higher education in social and behavioural sciences. Educational Research Review, 24, 1-9. https://doi.org/10.1016/j.edurev.2018.01.002

Clarà, M. (2015). What is reflection? Looking for clarity in an ambiguous notion. Journal of Teacher Education, 66(2015), 261-271. https://doi.org/10.1177/0022487114552028

Chen, N.S., Chun-Wang Wei, K., \& Liu, C.C. (2011). Effects of matching teaching strategy to thinking style on learner's quality of reflection in an online learning environment. Computers \& Education, 56(1). https://doi.org/10.1016/j.compedu.2010.08.021

Dewey, J. (1910). How we think. Boston, MA: D.C. Heath and Company.

Dyment, J., \& O'Connell, T. (2011). Assessing the quality of reflection in student journals: A review of the research. Teaching in Higher Education, 16, 81-97. doi: 10.1080/13562517.2010.507308

Fook, J., White, S., \& Gardner, F. (2006). Critical reflection: A review of contemporary literature and understandings. In S. White, J. Fook, \& F. Gardner (Eds.), Critical reflection in health and social care (pp. 1-18). Open University Press, Maidenhead.

Gibbons, M. (2002). The Self-directed learning Handbook: Challenging Adolescents Students to Excel. Wiley.

Goldberg, D.E., \& Sommerville, M. (2014). A whole new engineer (1st ed.). Douglas, MI: ThreeJoy Associates Inc.

Gün, B. (2011). Quality Self-reflection through Reflection Training. ELT Journal, 65.

Hoogerheide, V., \& Roelle, J. (2020). Example-based learning: New theoretical perspectives and useinspired advances to a contemporary instructional approach. Applied Cognitive Psychology, 34, 787-792. https://doi.org/10.1002/acp.3706

Kember, D., McKay, J., Sinclair, K., \& Yuet Wong, F.K. (2008) A four-category scheme for coding and assessing the level of reflection in written work. Assessment \& valuation in Higher Education, 33(4), 369-379.

Kori, K., Pedaste, M., Leijen, Ä., \& Mäeots, M. (2014). Supporting reflection in technology-enhanced learning. Educational Research Review, 11, 45-55. https://doi.org/10.1016/j.edurev.2013.11.003

Lee, H. (2005). Understanding and assessing preservice teachers' reflective thinking. Teaching and Teacher Education, 21(6), 699-715.

Leijen, Ä., Valtna, K., Leijen, D.A.J., \& Pedaste, M. (2012). How to determine the quality of students' reflections? Studies in Higher Education, 37(2), 203-217.

Poldner, E., Simons, P.R.J., Wijngaards, G., \& Van der Schaaf, M.F. (2012). Quantitative Content Analysis Procedures to Analyse Students' Reflective Essays: A Methodological Review of 
Psychometric and Edumetric Aspects. Educational Research Review, 7, 19-37.

http://doi:10.1016/j.edurev.2011.11.002.10.1016/j.edurev.2011.11.002

Rogers, R.R. (2001). Reflection in higher education: A concept analysis. Innovative Higher Education, 26(1), 37-57. https://doi.org/10.1023/A:1010986404527

Russell, T. (2005). Can reflective practice be taught? Reflective Practice, 6, 199-204.

Ruijter, K., \& Krab-Hüsken, L. (2012). Report for application for initial accreditation of the new Bachelor of Science degree (BSc) program; Limited program assessment. Honours program Technology and Liberal Arts \& Sciences. ATLAS The University College of the University of Twente.

Saks, S., \& Leijen, Ä. (2014). Distinguishing self-directed and self-regulated learning and measuring them in the E-learning Context. Procedia - Social and Behavioral Sciences, 112, 190-198.

Welch, M. (1999). The ABCs of reflection: A template for students and instructors to implement written reflection in service-learning. NSEE Quarterly, 25, 22-25.

Yost, D.S., Sentner, S.M., \& Forlenza-Bailey, A. (2000). An examination of the construct of critical reflection: Implications for teacher education programming in the 21 st century. Journal of Teacher Education, 51(1), 39.

Zimmerman, B.J. (2002). Becoming a Self-Regulated Learner: An Overview. Theory Into Practice, 41(2), $64-70$.

\section{APPENDIX: ATLAS REFLECTION GUIDE FOR THE SER}

Reflection is the act of thinking critically about an experience or critical incident in order to learn from that experience. Humans generally learn from experience on the basis of which they build mental models of the world. Subsequently, experience is understood in terms of these models. Sometimes, a learning experience will lead to a degree of "perplexity, hesitation, [or] doubt" (Dewey, 1910, p.9), leading a person to change his or her perspective or current views on the world. Then, mental models are adapted and understanding of the world becomes more sophisticated. Identifying, analysing and understanding the (personal) significance of such and experience is an act of higher-order (or: critical) reflection. These mental activities allow you to truly learn from experience.

In ATLAS, students reflect in their SER on their academic, professional and personal development. "Academic' relates to theoretical knowledge, "professional" refers to skills (from domain-specific skills to more domain general skills like leadership, communication or collaboration skills) and "personal" relates to e.g. beliefs, attitudes, norms, values, priorities and behaviors. It is expected that ATLAS students will have significant learning experiences or critical incidents in these areas from which they can learn, if they reflect on them.

\section{Finding a Critical Incident or Significant Learning Experience}

The first step is finding a critical incident or significant learning experience. This might take some time. Such an incident might be 1) non-routine, presenting an issue that required a (new) decision you were involved in, 2) a situation that required improvisation or innovation on your side, 3 ) a situation that required you to change a (common) procedure, or 4) one that was emotionally, physically or mentally demanding in some way (based on Kember, 2008). Think of as many of this kind of incidents that you can remember and make a list. Keep the list in mind and add new incidents if they come to mind over a few days.

The second step is to look over your list and make a choice for incidents to reflect upon. Choose learning experiences that fit the reflection themes (academic, professional, personal development). If you cannot find incidents that fit all three themes, explain what you did instead in your SER. Make sure you have at least three reflections. 


\section{Writing Your Reflections}

The third step is to write the reflections. For this, use the scaffold questions below (based on Ash \& Clayton, 2004). The answer to each of these questions (or some questions combined) could be a separate paragraph.

1) What did I learn?

2) How, specifically, did I learn it? (who, what, where, how?)

3) Why does this learning matter, or why is it significant?

4) In what ways will I use this learning; or what goals shall I set in accordance with what I have learned in order to improve myself, the quality of my learning, or the quality of my future experiences?

The first question addresses the learning. So, what is it that you learned? Needless to say, an answer to this question cannot be "a lot", "I learned so much", or "I grew as a person." These learnings are meaningless. Shortly and specifically describe what you learned.

The answer to the second question adds context to what you learned. Basically, it describes the critical incident. Zoom in and describe who was involved, what happened, where did it happen and how did it happen? You might want to combine the answers to question 1 and 2. The third question describes the significance of the learning. How or why does the learning matter to you? The final question addresses what the learning means for your further development. What will be different or will you do differently in the future now you had the experience? Describing the learning in terms of these questions results in a fullblown reflection.

\section{Feedback on the SER}

You will receive feedback on the quality of your reflection (and self-evaluation) in your SER. But what is a high-quality reflection? For this, a protocol used by Kember et al. (2008) was adapted to fit the purpose of providing feedback on the SER (see Table 1). The parameters that define the quality of the reflection are: 1) understanding of the critical incident in term of the student's learning (understanding dimension), 2) implication of the learning for other situations in which it might apply (transfer dimension), 3) the personal impact or - significance of the incident (personal dimension) and 4) the extent to which the incident has had implications for future goals or behaviours (future dimension). The highest level (described in the introduction), which is not common according to Kember et al. (2008), also includes evidence of a fundamental change in perspective (adaptation of mental models).

TABLE 1

\section{DESCRIPTION OF LEVELS OF REFLECTION}

\section{Level 1: Non- reflection}

The intended reflection shows no evidence of the student attempting to reach understanding of a critical incident or significant learning experience. The student is basically evaluating his or learning on a general level, the learning is not related to any other situation in which it might apply. The reflection is not personal, it could have been written by any student. No implications for future goals or behaviours mentioned.

\section{Level 2: Understanding}

A critical incident or learning experience is described to a certain extent, and the student is able to explain its meaning, but does not relate it to any other situation in which it might apply. Also, it does not become clear in what way the experience matters for the student personally, or how it affected future goals or behaviours.

\section{Level 3: Reflection}

A critical incident is described clearly, and the student is able to explain its meaning and how it relates to other situations in which it might apply. It is also clear how and why the experience has mattered to the student on a personal level. 
Level 4: Critical reflection

All of the above (level 3), while it is also clear how the incident has affected the student's future goals or behaviours. In addition, there is clear evidence of a change in perspective over a fundamental belief.

\section{Worked-Out Examples of Reflections on Different Levels}

Below are four examples of reflections on the levels explained in Table 1.

\section{Example \#1: Non-Reflection}

"This truly has been an amazing semester. The project and courses were all very interesting and we had to work really hard to make all the deadlines for the assignments and the deliverables. Sometimes, I thought I would fail, but I managed to stay motivated and in the end succeeded with help of my peers. I truly learned a lot and gained so much from this experience. I am looking forward to the next semester."

\section{Explanation}

No critical incidents described, general evaluation of the learning experience, not personal, no reference to other situations in which the learning applies, no future outlook and no evidence of a fundamental change in perspective.

\section{Example \#2: Understanding}

Me and my teammates frequently had discussions in which I was convinced of a certain direction we had to take, but my team mates just did not seem to get it. We then talked for hours and hours and we got nowhere. I kind of understood their ideas, but I guess I learned that in a team being right does not mean it will thus happen in the best way possible.

\section{Explanation}

Critical incident somewhat clear (but still general) and understanding emerging, but not yet very personal, no reference to other situations in which the learning applies, no future outlook and no evidence of a fundamental change in perspective.

\section{Example \#3: Reflection}

In social science, I learned that even the most obvious things are not always obvious. In my first essay, I wrote that everybody human being likes music and based my further reasoning on that premise. Obvious to me, but the feedback stated: "What is the evidence for this?" It irritated me, why did I need to explain that? But then I received some readings that contained evidence that there are also people that aren't into it at all and that there is quite some variation in this phenomenon. I began to doubt every premise that sprouted from my prior knowledge and realized I should have been more aware of my personal premises being correct or not in any of my writings and discussions I was involved in this semester.

\section{Explanation}

Critical incident clearly described, understanding of the learning is clear and the reflection is personal. Also, there is reference to other situations in which the learning applies, but no clear future outlook and no evidence of a fundamental change in perspective. 


\section{Example \#4: Critical Reflection}

Being a math and physics person, I guess I learned in school that there is a solution to every problem. I could not think of it in any other way and I became good at it. Teachers always complimented me on my elegant and simple solutions. But I learned that for the real problems there are no easy solutions. I tried to approach the semester project in my old way and tried to come up with a simple and elegant solution. However, once I thought I had it, someone would come up with a perspective I did not think of and we had to start all over. This was really frustrating to me, these problems were just unsolvable, so why should I even bother giving it a try? But during a project lecture I learned about the concept of mitigation. Some problems are so complex that it is impossible to find a generic solution, you just have to accept that what you can do, is see where you can have the most impact. This realization gave me new confidence that, although I maybe cannot find generic solutions, there is always something I CAN do. I think this applies to many problems that I will face as a New Engineer, I see now. I just need (and want!) to learn as much as I can about problem solving in different disciplines to be able to face the complexity that is inherent to the problems that world is facing today and I will be working on in the future.

\section{Explanation}

Critical incident clearly described, understanding of the learning is clear and the reflection is personal. Also, there is reference to other situations in which the learning applies, and an outlook to future goals and behaviours. Also, there is evidence of a change in perspective.

\section{Closing Remarks on Reflection in Your SER}

An ATLAS student should at least be able to write level 3 reflection, one in each of the three reflection areas (academic, professional, personal development). Use the scaffold questions to write your reflections and then self-check whether your reflections are at the right level (3 or 4). If not, assess to what extent your reflection can be improved along the four dimensions (understanding, transfer, personal and future dimension). 